Louisiana State University

LSU Digital Commons

Faculty Publications

Department of Physics \& Astronomy

3-1-2014

\title{
What will it take to observe processes in 'real time'?
}

\author{
Stephen R. Leone \\ University of California, Berkeley \\ C. William Mccurdy \\ University of California, Berkeley \\ Joachim Burgdörfer \\ Technische Universität Wien \\ Lorenz S. Cederbaum \\ Universität Heidelberg \\ Zenghu Chang \\ University of Central Florida
}

See next page for additional authors

Follow this and additional works at: https://digitalcommons.Isu.edu/physics_astronomy_pubs

\section{Recommended Citation}

Leone, S., Mccurdy, C., Burgdörfer, J., Cederbaum, L., Chang, Z., Dudovich, N., Feist, J., Greene, C., Ivanov, M., Kienberger, R., Keller, U., Kling, M., Loh, Z., Pfeifer, T., Pfeiffer, A., Santra, R., Schafer, K., Stolow, A., Thumm, U., \& Vrakking, M. (2014). What will it take to observe processes in 'real time'?. Nature Photonics, 8 (3), 162-166. https://doi.org/10.1038/nphoton.2014.48

This Article is brought to you for free and open access by the Department of Physics \& Astronomy at LSU Digital Commons. It has been accepted for inclusion in Faculty Publications by an authorized administrator of LSU Digital Commons. For more information, please contact ir@lsu.edu. 


\section{Authors}

Stephen R. Leone, C. William Mccurdy, Joachim Burgdörfer, Lorenz S. Cederbaum, Zenghu Chang, Nirit Dudovich, Johannes Feist, Chris H. Greene, Misha Ivanov, Reinhard Kienberger, Ursula Keller, Matthias F. Kling, Zhi Heng Loh, Thomas Pfeifer, Adrian N. Pfeiffer, Robin Santra, Kenneth Schafer, Albert Stolow, Uwe Thumm, and Marc J.J. Vrakking 


\section{Lawrence Berkeley National Laboratory}

\section{Recent Work}

\section{Title}

What will it take to observe processes in 'real time'?

Permalink

https://escholarship.org/uc/item/7jr9h6zv

Journal

Nature Photonics, 8(3)

ISSN

1749-4885

Authors

Leone, SR

Mccurdy, CW

Burgdörfer, J

et al.

Publication Date

2014-03-01

\section{DOI}

10.1038/nphoton.2014.48

Peer reviewed 


\title{
What will it take to observe processes in 'real time'?
}

\author{
Stephen R. Leone, C. William McCurdy, Joachim Burgdörfer, Lorenz S. Cederbaum, Zenghu Chang, \\ Nirit Dudovich, Johannes Feist, Chris H. Greene, Misha Ivanov, Reinhard Kienberger, Ursula Keller, \\ Matthias F. Kling, Zhi-Heng Loh, Thomas Pfeifer, Adrian N. Pfeiffer, Robin Santra, Kenneth Schafer, \\ Albert Stolow, Uwe Thumm and Marc J. J. Vrakking
}

\section{Even for simple systems, the interpretations of new attosecond measurements are complicated and provide only a glimpse of their potential. Nonetheless, the lasting impact will be the revelation of how short-time dynamics can determine the electronic properties of more complex systems.}

A ttosecond science is still in its infancy, yet impressive results and discoveries are already emerging ${ }^{1}$. Experiments have provided information about electron ejection from orbitals with timescales that differ by as little as tens of attoseconds, allowing valence-electron wave packets to be characterized. Scientists are now able to manipulate and steer electrons using laser fields, allowing them to probe recollision physics and ionization dynamics on attosecond timescales.

However, formidable challenges have arisen that counterbalance the promise of these early successes. A strong probe light field can modify the potential surfaces and alter the dynamics one is trying to observe. As most measurements are performed in a complicated temporal regime in which two pulses overlap, simplified descriptions of the time-dependent wavefunction are insufficient. Most importantly, there is an urgent need to separate processes driven or altered by the various probing light fields from the natural time dynamics one is seeking to measure.

\section{Fundamental issues for}

attosecond science. In principle, attosecond science reveals information about electron correlations $s^{2,3}$, particularly strong correlations, through innovative experiments and theory that involve time, phase, interferences and angular distributions. The availability of $\sim 100$ as pulses in the extreme ultraviolet (XUV) spectral region ${ }^{1}$ has allowed many new experimental and theoretical methods to be developed, enabling numerous new results to be obtained. Examples include attosecond streaking for measuring the apparent time delays of electrons born

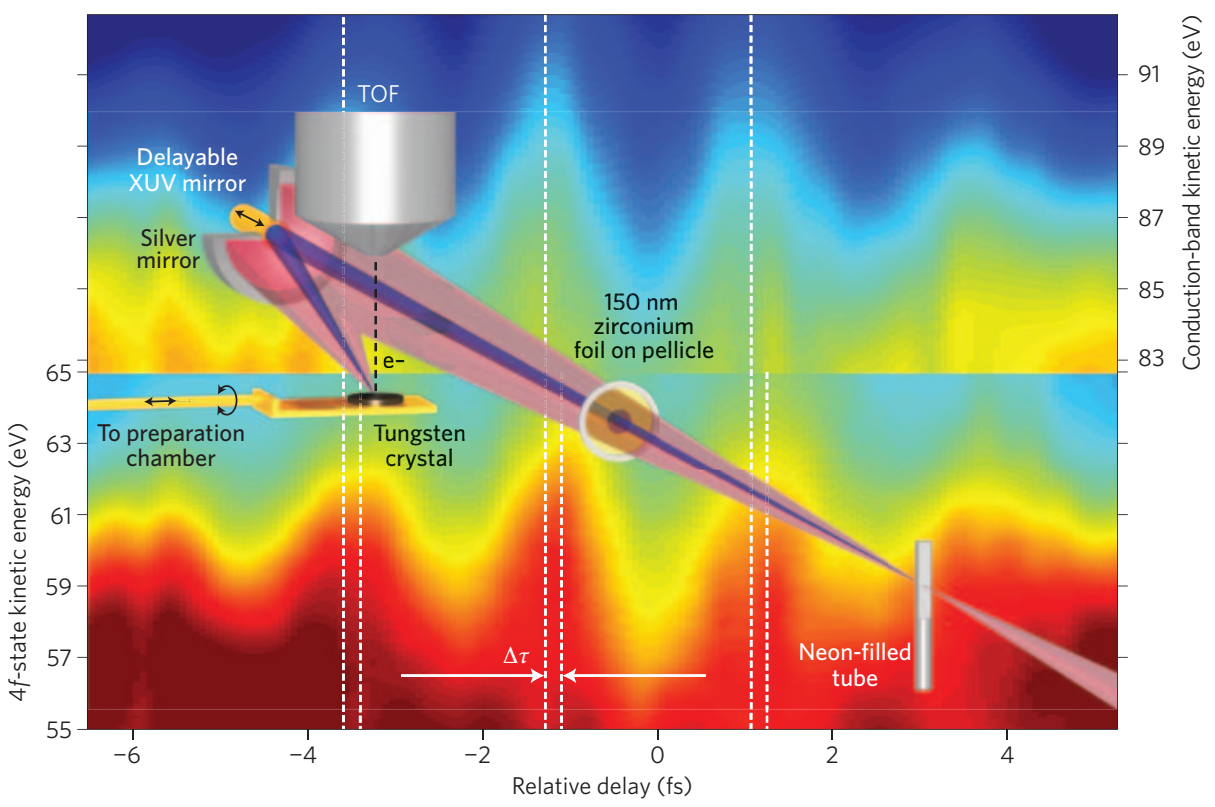

Figure 1 | Streak field measurements of surface photoelectron ejection. The apparatus for streaking of electrons from a tungsten surface is shown overlaid on streak traces from the conduction-band electrons and $4 f$ electrons of $W(110)$. The attosecond pulses are generated in the neon-filled tube, producing the inner XUV beam, which is time delayed from the outer nominal $800 \mathrm{~nm}$ pulse, separated by the zirconium foil. The streak traces indicate a 110 as delay, with the conduction-band electrons (upper) arriving first. TOF, time of flight. Figure reproduced with permission from ref. 13, (c) 2007 NPG.

into a laser field from different states, attosecond transient absorption for investigating strong-field ionization and coherence phenomena, and attosecond tunnelling and recollision spectroscopy ${ }^{1,4}$

The advent of many new methods for extracting fundamental information on attosecond timescales has brought even the most basic concepts under scrutiny. Moreover, researchers must push the limits of time dynamics in the unfamiliar regime of XUV photon energies, which exceed ionization limits and for which the potential surfaces for core-hole excited states are largely unknown. Major challenges for the field include identifying the observables accessed by a specific experimental set-up and designing experiments to address these observables.

What can present and future sources and methods measure? There is a broad 
consensus that attosecond measurements are constrained by currently available techniques. Isolated attosecond (70-500 as) pulses and attosecond pulse trains have been reliably produced for photon energies in the approximate range $15-120 \mathrm{eV}$ using the process of high-harmonic generation ${ }^{1}$. This process also serves as the basis for attosecond recollision experiments. An ideal experiment would combine two isolated attosecond pulses: one for pump excitation and one as a time-delayed probe. However, the limited fluxes that can currently be generated using attosecond pulses combined with typical absorption cross-sections make it difficult to perform true attosecond-pump-attosecond-probe experiments. In the future, higher-flux lasers with ultrabroadband spectra and better high-harmonic conversion schemes, along with free-electron lasers, will undoubtedly produce sufficiently high fluxes of isolated attosecond pulses for attosecond-pump-attosecond-probe experiments.

Until now, experiments employing isolated attosecond pulses have often combined one attosecond pulse with a carrier-envelope-phase stabilized ${ }^{1}$, fewcycle $\sim 5 \mathrm{fs}$ infrared pulse. As a result, experiments requiring subfemtosecond resolution must be performed in the difficult regime of temporal overlap between a short pulse and a longer one. Intriguing results have been obtained, but theoretical analysis of even the simplest experiments still requires considerable advances to understand the pulse overlap region and the influence of the combined, and often strong, fields.

Time-resolved experiments on complex targets, such as biomolecules, nanoparticles and solid surfaces, will be rewarding, but their analysis is challenging, because accurate theoretical models are needed for the strong transient distortion of electronic structures in ultrashort intense pulses of radiation.

Additional significant advances will be made when true attosecond-pumpattosecond-probe experiments are realized, although these experiments will be performed on unfamiliar high-energy states for which methods to calculate the potential energy surfaces of core hole excited states will require further development.

In addition, highly differential measurements are important for the field to advance - for example, coincidence detection of angular, spectral, mass and time information ${ }^{5}$ and multidimensional X-ray spectroscopies ${ }^{6}$, which can potentially prepare and probe valence-

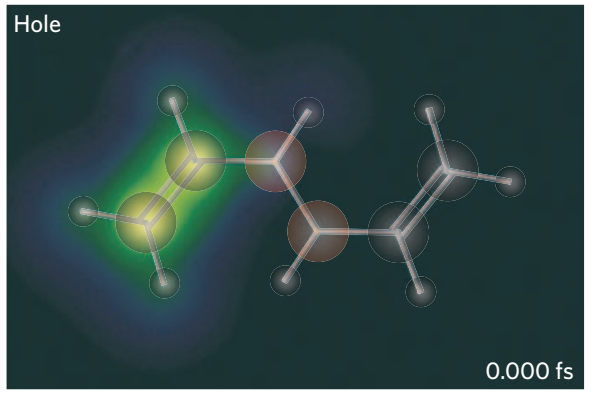

diffractive imaging. Such methods have the potential to provide images of complex chemical transformations, charge migration and plasmonic charge motion in nanostructures. However, they have complications, such as requiring high $\mathrm{X}$-ray fluxes and intensities. Furthermore, some of the basic assumptions made for static $\mathrm{X}$-ray scattering experiments may have to be modified in a nontrivial manner when the electrons in the target molecule are in nonstationary states (superposition states).

Quantum electrodynamics, which describes light in terms of particles (photons), is essential for analysing timeresolved X-ray scattering patterns. This is because semiclassical theory, which describes light in terms of classical waves, does not incorporate inelastic scattering and incorrectly predicts that the diffraction pattern relates to the instantaneous positions of the electrons. Attosecondresolved X-ray scattering patterns from electron wave packets may be better interpreted if the techniques of X-ray phase-contrast imaging ${ }^{8}$ can be employed.

Does attosecond science measure a process in real time? Time is not a quantum-mechanical observable, rather it is something the experimentalist accesses by, for example, using a pump-probe delay $^{9}$. However, some observables, such as tunnelling times or arrival-time distributions, characterize the duration of specific processes. These observables are often associated with phase shifts. Unfortunately, and perhaps ironically, not every type of measurable phase shift provides time information. A deep understanding of attosecond processes is inextricably linked to distributions of times of arrival, detailed phase-shift information and interferences between channels.

electron wave packets with an attosecond time resolution.

\section{'Listening to electrons'. Because} many experimental methods observe changes (such as spectroscopic corelevel transitions ${ }^{7}$ and electron ejection ${ }^{1}$ ) indirectly through 'reporter' atoms or electrons, the outcomes are like 'listening' to the effect the electrons or holes have on various sites, rather than directly observing electron motion. Such techniques exhibit remarkable sensitivity to changes in the electronic state, oxidation state, charge, chemical environment and even bond lengths of a particular atom. Experiments that indirectly monitor chemical changes are currently difficult to interpret. These approaches may transition eventually to attosecond experiments that utilize attosecond excitation and coherent
Such measurements have the additional complication of the need to determine whether the observed phase shifts give the desired time information.

Relating results to a classical picture often provides a physical relationship to be correlated with the timescale of an event. Rigorous analysis of attosecond measurements by theory can confirm that the observed timescale (for example, the timescale related to the net phase accumulation) does provide new physical insights into the collective electronic dynamics of complex quantum systems. ejection. One of the major successes for attosecond science has been the measurement of the delays of electrons that enables an experimental observable

\section{The success of time-resolved electron}


ejected from atoms in the gas phase and from solid-state surfaces upon direct ionization or Auger decay. This method, called streakfield detection ${ }^{1}$, has been refined to detect differences in electron ejection times of the order of tens of attoseconds. In the streak-field method, a strong field from a near-infrared few-cycle pulse is used to shift the momentum of an electron released into a laser field, providing exquisite time resolution for the time of birth of the electron. In $\mathrm{Ne}$ atoms, electron emission from the $2 p$ orbital was observed to have a $21 \pm 5$ as delay relative to that from the $2 s$ orbital for $100 \mathrm{eV}$ photons ${ }^{10}$. Numerous high-level theoretical investigations have been unable to reproduce quantitatively the experimentally observed delay; instead, they underestimate the delays by a factor of at least two. Despite this discrepancy, tremendous advances have been made in our theoretical understanding of the distinct contributions to the delay times in atomic systems, including those of the initial-state polarization, the Coulomb-laser coupling in the final continuum state and the interaction of the electron with its entangled ion state ${ }^{11,12}$.

Surface-electron escape times. Initial measurements ${ }^{13}$ for $100 \mathrm{eV}$ photons on a clean tungsten surface showed that $4 f$ electrons escape with a time delay of 110 as relative to conduction-band electrons (Fig. 1). Related experiments are currently being performed for other surfaces and layered systems and for different excitation energies; the delay times for these measurements are expected to differ markedly from those for a clean tungsten surface. By selectively adding monolayers, it should be possible to obtain information about the mechanisms that determine the timescale for electron escape.

Theoretical studies have suggested that there are multiple contributions to the delays in these surface-electron escapetime measurements ${ }^{14}$; these contributions include the image charge potential, the screening response of surface layers to the streaking field, the degree of localization of the initial-state wavefunctions, the finalstate band structure at high energies and inelastic scattering processes.

Although the interpretation of surfaceelectron emission delays is still in its infancy, the ability of the surface streaking method to obtain attosecond delay times raises the possibility of performing other measurements on strongly correlated electron materials. For example, streaking measurements of electron ejection could be performed on superconducting films and metal-to-insulator transition materials, which would allow electron emission delay

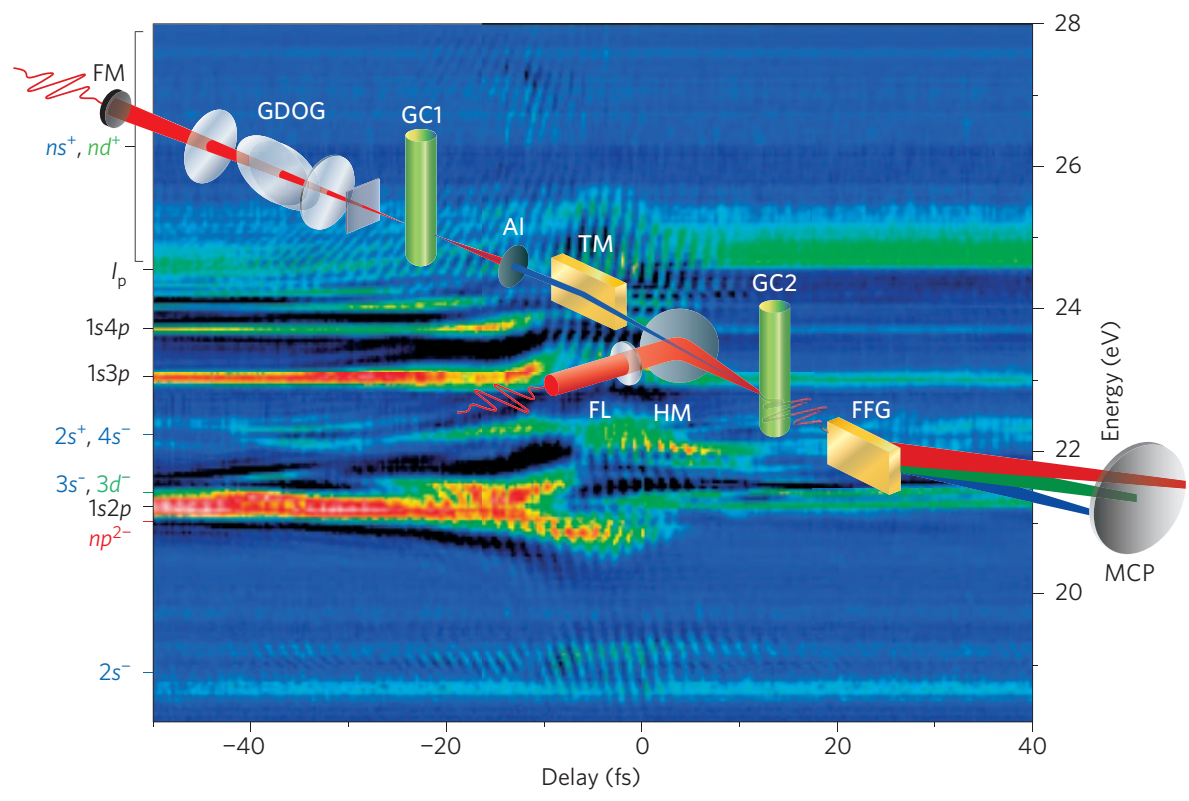

Figure 3 | Transient absorption measurements on $\mathrm{He}$, taking advantage of the polarization of the medium. The apparatus for attosecond transient absorption (FM, focusing mirror; GDOG, generalized double optical gating optics; GC1, argon or xenon-backed gas cell for attosecond pulse generation; $\mathrm{Al}$, aluminium foil filter; TM, toroidal mirror; FL, focusing lens; HM, hole-drilled recombination mirror; GC2, heliumbacked gas cell for absorption measurements; FFG, flat-field XUV grating; MCP, microchannel plate and phosphor screen detector) is shown overlaying experimental data for $730 \mathrm{~nm}$ field manipulation of the polarization probed by an isolated attosecond pulse in $\mathrm{He}$ atoms, revealing light-induced states and subcycle oscillations due to the few-cycle $730 \mathrm{~nm}$ field. Figure reproduced with permission from ref. 20, (c) 2013 NPG.

times to be assessed above and below the critical transition temperatures for the first time. The use of attosecond science to characterize the ground-state properties of strongly correlated electron materials is potentially a major addition to the arsenal of methods for measuring and analysing electron correlation in solids.

Direct absorption probing of atomic dynamics. The combination of an XUV attosecond pulse with a stronger femtosecond pulse that comes before or after it can provide time-resolved spectral information. This information is imprinted onto the attosecond pulse and can be measured by transient absorption. Transient absorption is a pump-probe technique that is applicable to gases, liquids and solids; it is well matched to the extremely broad bandwidth of isolated attosecond pulses. Probing with an XUV or X-ray attosecond pulse provides timeresolved chemical information for complex systems by core-level spectroscopic shifts.

The first attosecond transient absorption experiment was performed on the strongfield ionization of $\mathrm{Kr}$ atoms ${ }^{15}$, by using an $800 \mathrm{~nm}$ pulse to ionize $\mathrm{Kr}$ atoms and an isolated attosecond pulse to subsequently detect $\mathrm{Kr}^{+}, \mathrm{Kr}^{2+}$ and $\mathrm{Kr}^{3+}$ ions. A valence orbital spin-orbit electronic wave packet in $\mathrm{Kr}^{+}$was observed and its degree of coherence was determined.

Electronic coherences have now been observed in the higher ionization states of atoms produced by strong-field ionization. The attosecond transient absorption method provides a means for performing new measurements of charge state dynamics, state-resolved processes and electronic superpositions, especially in molecules.

Complications occur when the pump and probe pulses overlap. As the transient absorption method inherently measures the effect of the combined light fields over the lifetime of the core-hole-excited probe (final) state, and not the instantaneous values of the fields, it produces a spectral measurement that is nonlocal in time ${ }^{15}$. Phase shifts arising from the strong dipole coupling between the ground and excited states by the near-infrared field induce a transient line shift in the XUV spectrum when the pump and probe pulses overlap ${ }^{16}$.

Charge migration in molecules - a central test for attosecond dynamics. Using strong-field ionization or direct attosecond 
ionization, it is possible to produce localized hole states in migrating molecules driven by electron correlation. Theory shows that a valence hole localized in one part of a molecule can migrate rapidly across the molecule (Fig. 2) ${ }^{17}$, because it is actually a superposition of a large number of valence excited states; it can even return in time to the initial hole configuration. Timeresolved chemical shifts of reporter atoms can be used to follow this charge migration. Such results raise fundamental questions about inducing electron dynamics via attosecond or strong-field photoionization, such as whether the nonstationary state of the parent ion can be described by a Schrödinger wavefunction without including the photoelectron and what role decoherence due to nuclear motion plays.

The interactions between the photoelectron and the parent ion trigger many-body effects ${ }^{18}$ that enhance the entanglement between the photoelectron and the ion, leading to electron wave packet dynamics within the ion. Such effects will most likely be investigated by attosecond methods in the near future. When combined with decoherence induced by nuclear motion, these methods will finally enable charge migration to be probed in real time.

\section{How separable are nuclear motion and} electronic dynamics? Nuclear motion is usually considered to occur on a much longer timescale than electron dynamics, but the ability to separate these two timescales is not always certain. The outcome of an attosecond electron dynamics measurement in molecules can still be influenced by the very nuclear motions that one wishes to avoid ${ }^{19}$. A state change during a passage through a conical intersection may occur in 10-100 fs in some cases, but non-Born-Oppenheimer processes may be evident even within a fraction of a vibrational period, perhaps as fast as a few femtoseconds. Probing electronic motion during the actual change of electronic state with attosecond pulses promises to reveal details of nonadiabatic transitions that will test the limits of the current theoretical understanding. Herein lies both the excitement and the challenge of attosecond measurements in highly excited complex systems.

\section{Revealing information via polarization.}

In attosecond transient absorption, an isolated attosecond pulse can be used to create a polarization of the medium, and the time-evolving polarization can then be probed by perturbing the system with a few-cycle near-infrared or visible pulse (Fig. 3) ${ }^{20}$. The near-infrared pulse may affect the polarization of the medium by ionizing it or by coupling to other states, thereby creating Autler-Townes doublets, electromagnetically induced transparency or Mollow triplets and providing the possibility of measuring electronic superpositions and lifetimes of autoionizing and Auger-decaying states. The near-infrared pulse has also been used to impart a specific phase to the polarization, modifying a Lorentzian line shape into a Fano profile, and vice versa ${ }^{21}$.

\section{Solid-state insulator-to-conductor} transition. A surprising and rapid transformation of a dielectric material, silicon dioxide (fused silica), from an insulator to a conductor, has been observed by attosecond transient absorption ${ }^{22}$. With a 4 fs few-cycle, near-infrared laser field in the several volts per ångström range, reversible insulator-to-conductor dynamics is observed on subcycle timescales. These results presage possible successes of experiments on metal-toinsulator transitions in strongly electroncorrelated materials, exciton dynamics and bandgap renormalization.

\section{Strong-field attosecond control of electron tunnelling and recollision.} Electron recollision was the first attosecond timescale process to capture the imagination of many scientists worldwide $^{3,4}$. In a semiclassical model, an oscillating near-infrared laser field can cause an electron to be ejected from an atom by tunnelling through the Coulomb and laser-field potential, reversing the electron direction and causing the electron to undergo a recollision. This process, known as high-harmonic generation, provides a unique probe via light emission. Alternatively, the tunnelling trajectories and timescale of the outgoing electron may be observed, as can abovethreshold ionization, double ionization and excitation. This approach opens new avenues for both measurement and control: it can reveal the remarkable physics that occurs on tunnelling timescales, and be used to create and manipulate the shape of attosecond pulses. This area enables attosecond-timescale control of atomic and molecular properties.

Strong-field recollision and ionization phenomena have been used in other major advances ${ }^{4}$. Application of angular-resolved measurements with nearly circularly polarized light provides an 'attoclock' assessment ${ }^{23}$ of the timescale for strongfield electron tunnelling. It is possible to image Dyson molecular orbitals through high-harmonic emission, to decode the hole motion in the time between ionization and recombination ${ }^{24}$, to follow the dynamic evolution of bonds, and to probe core electron excitation and elastic and inelastic scattering of the electron.

Each of the possible events in the third step of the recollision process has its own advanced theoretical interpretation ${ }^{3}$. Electron correlation phenomena are ubiquitous in the strong fields used to interrogate multielectron systems ${ }^{3}$. Not only does the highest occupied molecular orbital participate in recombination and high-harmonic emission, but the next lower lying orbitals, which are electronically excited ion states, are also involved in the recombination of the recolliding electron.

It is conceivable to ask whether the migration of a hole created by an attosecond pulse might be probed by field manipulation of a recolliding electron. This will be difficult to achieve in complex systems, but encouraging work on laserinduced electron diffraction by recolliding electrons ${ }^{25}$ has begun to reveal pictures of a vibrating diatomic molecule.

Outlook. Like other time-resolved measurements, attosecond measurements are intrinsically indirect probes of time, but they have the strong potential to address electron dynamics timescales - not simply internuclear dynamics. There is good evidence to suggest that scientists are correctly interpreting attosecond processes in terms of the real observables, such as phase-shift information, distributions of arrival times and spectral shifts in reporter atoms, even for complicated systems. Researchers will eventually obtain attosecond time-resolved movies of electron charge migration, and there is an excellent chance we will soon start to understand charge migration in large molecules by using existing attosecond methods.

There is a clear consensus that in the first decade of attosecond science, experiments were limited to using strong near-infrared fields or combined attosecond and femtosecond pulses because of the low intensity of available attosecond pulses. Nevertheless, remarkably rapid progress has been made in this new time domain. The field will evolve as attosecond pulses with sufficiently high fluxes are produced and free-electron lasers become available, surmounting a principal experimental barrier, covering more wavelength ranges and permitting attosecondpump-attosecond-probe measurements. Nonlinear X-ray methods will be developed to access valence energies and processes of interest ${ }^{6}$. Coincidence methods ${ }^{5}$ will 
enhance these measurements by adding multidimensionality to the mix, providing new parameters to unravel more-complex systems. Although the attosecond field is more subtle than the already challenging picosecond and femtosecond time regimes, experiments have established and theories have confirmed multiple successes. Consequently, confidence is growing that more and reliable attosecond time-resolved measurements on unknown and complex systems will be made in the future.

Stephen R. Leone ${ }^{1,2 *}$, C. William McCurdy ${ }^{2,3}$, Joachim Burgdörfer ${ }^{4}$, Lorenz S. Cederbaum ${ }^{5}$, Zenghu Chang ${ }^{6}$, Nirit Dudovich ${ }^{7}$ Johannes Feist ${ }^{8}$, Chris H. Greene ${ }^{9}$, Misha Ivanov ${ }^{10,11,12}$, Reinhard Kienberger ${ }^{13,14}$, Ursula Keller ${ }^{15}$, Matthias F. Kling ${ }^{13,16}$, Zhi-Heng Loh ${ }^{17}$, Thomas Pfeifer ${ }^{18,19}$, Adrian N. Pfeiffer ${ }^{1,2,20}$, Robin Santra ${ }^{21,22}$, Kenneth Schafer ${ }^{23}$, Albert Stolow ${ }^{24,25,26}$, Uwe Thumm ${ }^{27}$ and Marc J. J. Vrakking ${ }^{10}$ are at ${ }^{1}$ Departments of Chemistry and Physics, University of California, Berkeley, California 94720, USA. ${ }^{2}$ Ultrafast $X$-Ray Science Laboratory, Chemical Sciences Division, Lawrence Berkeley National Laboratory, University of California, Berkeley, California 94720, USA. ${ }^{3}$ Department of Chemistry, University of California, Davis, California 95616, USA. ${ }^{4}$ Institute for Theoretical Physics, Vienna University of Technology, 1040 Vienna, Austria. ${ }^{5}$ Theoretische Chemie, Ruprecht-Karls-Universität, D69120 Heidelberg, Germany. ${ }^{6} \mathrm{CREOL}$ and Department of Physics, University of Central Florida, Orlando, Florida 32816, USA. ${ }^{7}$ Department of Physics and Complex Systems, Weizmann Institute of Science, Rehovot 76100, Israel. ${ }^{8}$ Departamento de Física Teórica de la Materia Condensada, Universidad Autónoma de Madrid, E-28049 Madrid, Spain. ${ }^{9}$ Department of Physics, Purdue University, West Lafayette, Indiana 47907,
USA. ${ }^{10}$ Max Born Institute, Max Born Strasse 2A, 12489 Berlin Adlershof, Berlin, Germany. ${ }^{11}$ Department of Physics, Humboldt University, Newtonstrasse 15, 12489 Berlin, Germany. ${ }^{12}$ Department of Physics, Imperial College London, South Kensington Campus, SW7 2AZ London, UK. ${ }^{13}$ Max-Planck-Institut für Quantenoptik, Hans-Kopfermann-Strasse 1, Garching 85748, Germany. ${ }^{14}$ Department of Physics, Technische Universität München, James Franck Strasse, Garching 85748, Germany. ${ }^{15}$ Physics Department, ETH Zurich, 8093 Zurich, Switzerland. ${ }^{16}$ Physics Department, Ludwig-Maximilians-Universität München, Am Coulombwall 1, 85748 Garching, Germany. ${ }^{17}$ Division of Chemistry and Biological Chemistry and Division of Physics and Applied Physics, School of Physical and Mathematical Sciences, Nanyang Technological University, S637371 Singapore. ${ }^{18}$ Max-Planck-Institut für Kernphysik, 69117 Heidelberg, Germany. ${ }^{19}$ Center for Quantum Dynamics, RuprechtKarls-Universität, Heidelberg, 69120 Heidelberg, Germany. ${ }^{20}$ Institute for Optics and Quantum Electronics, Friedrich Schiller University Jena, Max-Wien-Platz 1,07743 Jena, Germany. ${ }^{21}$ Center for Free-Electron Laser Science, DESY, Notkestrasse 85, D-22607 Hamburg, Germany. ${ }^{22}$ Department of Physics, University of Hamburg, Jungiusstrasse 9, D-20355 Hamburg, Germany. ${ }^{23}$ Department of Physics and Astronomy, Louisiana State University, Baton Rouge, Louisiana 70803, USA. ${ }^{24}$ Department of Physics, University of Ottawa, Ottawa Ontario K1N 6N5, Canada. ${ }^{25}$ Departments of Chemistry and Physics, Queen's University, Kingston, Ontario K7L 3N6, Canada. ${ }^{26}$ SDT, Emerging Technologies Division, National Research Council of Canada, 100 Sussex Drive, Ottawa, Ontario K1A OR6, Canada. ${ }^{27} \mathrm{~J}$. R. Macdonald Laboratory, Department of Physics, Kansas State University, Manhattan, Kansas 66506, USA.

*e-mail: srl@berkeley.edu
References

1. Krausz, F. \& Ivanov, M. Rev. Mod. Phys. 81, 163-234 (2009).

2. Sansone, G., Pfeifer, T., Simeonidis, K. \& Kuleff, A. I. ChemPhysChem 13, 661-680 (2012).

3. De Morisson Faria, C. F. \& Liu, X. J. Mod. Opt. 58, 1076-1131 (2011).

4. Haessler, S., Caillat, J. \& Salières, P. J. Phys. B 44, 203001 (2011)

5. Schmidt-Böcking, H. et al. Nucl. Instrum. Methods Phys. Res. B 233, 3-11 (2005).

6. Mukamel, S., Healion, D., Zhang, Y. \& Biggs, J. D. Annu. Rev Phys. Chem. 64, 101-127 (2013).

7. Loh, Z.-H. \& Leone, S. R. J. Phys. Chem. Lett. 4, 292-302 (2013).

8. Dixit, G., Slowik, J. M. \& Santra, R. Phys. Rev. Lett. 110, 137403 (2013).

9. Muga, J. G., Mayato, R. S. \& Egusquiza, I. L. Time in Quantum Mechanics, Springer Lecture Notes in Physics Vol. 734, 2nd edn (Springer, 2008).

10. Schultze, M. et al. Science 328, 1658-1662 (2010).

11. Pazourek, R., Feist, J., Nagele, S. \& Burgdörfer, J. Phys. Rev. Lett. 108, 163001 (2012).

12. Zhang, C.-H. \& Thumm, U. Phys. Rev. A 82, 043405 (2010).

13. Cavalieri, A. L. et al. Nature 449, 1029-1032 (2007).

14. Lemell, C., Solleder, B., Tökési, K \& Burgdörfer, J. Phys. Rev. A 79, 062901 (2009).

15. Goulielmakis, E. et al. Nature 466, 739-743 (2010).

16. Pabst, S. et al. Phys. Rev. A 86, 063411 (2012).

17. Dutoi, A. D., Wormit, M. \& Cederbaum, L. S. J. Chem. Phys. 134, 024303 (2011).

18. Pabst, S., Greenman, L., Ho, P. J., Mazziotti, D. A. \& Santra, R. Phys. Rev. Lett. 106, 053003 (2011).

19. Kraus, P. M. et al. Phys. Rev. A 85, 043409 (2012).

20. Chini, M. et al. Sci. Rep. 3, 1105 (2013).

21. Ott, C. et al. Science 340, 716-720 (2013).

22. Schultze, M. et al. Nature 493, 75-78 (2013).

23. Pfeiffer, A. N., Cirelli, C, Smolarski, M. \& Keller, U. Chem. Phys. 414, 84-91 (2013).

24. Smirnova, O. et al. Nature 460, 972-977 (2009).

25. Blaga, C. I. et al. Nature 483, 194-197 (2012).

\section{Acknowledgements}

This Commentary evolved from the presentations and discussions at the workshop "Unraveling the Interpretations of Attosecond Measurements" held in March 2013 in Washington, DC, sponsored by the Council on Chemical and Biochemical Sciences of the U.S. Department of Energy, Office of Science, Office of Basic Energy Sciences. The authors are grateful to the members of the Council for their encouragement and assistance in developing this workshop. In addition, the authors acknowledge the agencies that provided funding for individual research programs in attosecond and related science, without which this workshop and Commentary would not have been possible. 